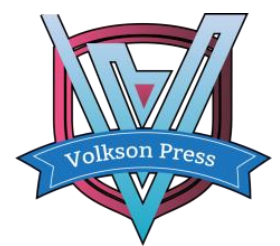

Contents List available at VOLKSON PRESS

Intelligent Computing and Information Engineering (ICIE )

DOI : http://doi.org/10.26480/icie.01.2017.36.40

Journal Homepage: : https://www.intelcomp-design.com/

\title{
A Reasoning Algorithm Based on Novel Extension Rule in Wumpus World
}

Cai Zhennan¹, Gao Ge¹, Han Zijian¹, Zhu Kangning11, Li Guangli², Lü Shuai²*

${ }^{1}$ College of Software, Jiliin University, ChangChun 130012, China

${ }^{2}$ College of Computer Science and Technology, Jiliin University, ChangChun 130012, China

*lus@jlu.edu.cn

This is an open access article distributed under the Creative Commons Attribution License, which permits unrestricted use, distribution, and reproduction in any medium, provided the original work is properly cited

\section{ARTICLE DETAILS}

\section{Article History:}

Received 12 May2017

Accepted 12 July 2017

Available online 14 September 2017

Keywords:

Artificial intelligence; Automatic reasoning; Novel Extension Rule; Wumpus World.

\section{ABSTRACT}

This paper presents a new algorithm based on Novel Extension Rule for reasoning problems in Wumpus World. This algorithm describes these problems by propositional logic terms and solves them with Novel Extension Rule. In Wumpus World, the algorithm ascertains possible positions with pits or the wumpus in order to find a safe way to the gold. Experimental results prove its soundness and completeness.

\section{Introduction}

With the development of the artificial intelligence, robots begin to function like human beings in multiple ways. Cognitive robots are those robots that have high-level cognitive capability of reasoning, which can adapt to the complex environment and accomplish complex tasks [1-3]. Based on the idea of cognitive reasoning, the robots can study, self-develop and make decisions. Many scholars have made research results in the cognitive robot, such as Chidori's bioinspired applications, GONG Wei-wei and others' hierarchical framework of robotic system etc [4,5]. The theorem proving method based on Extension Rule was provided on Journal of Automated Reasoning, solved the problem that resolution reasoning has with high complementary factor [6]. After continuous developing and improving, Lin Hai provided Extension Rule, the inverse of resolution, and a theorem proving algorithm based on Extension Rule called ER [6]. Sun, J.G. provided a new algorithm called NER based on Extension Rule [7]. The NER algorithm decides whether a clause set is satisfiable by deciding one literal set includes another one or not, while ER algorithm decides it by counting the number of maximum terms expended. Extension rule has become an important reasoning method [8].

This paper applies NER to solve reasoning problems in Wumpus World and examples show agents how to select right actions based on the environment parameters [9].

\section{THE WUMPUS WORLD}

\subsection{Introduction of The Wumpus World}

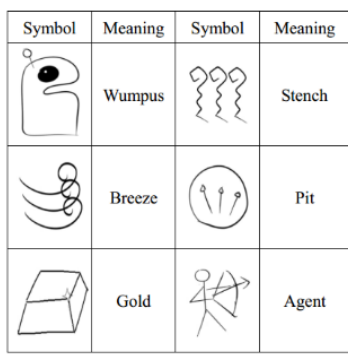

Figure 1: A map of the Wumpus World

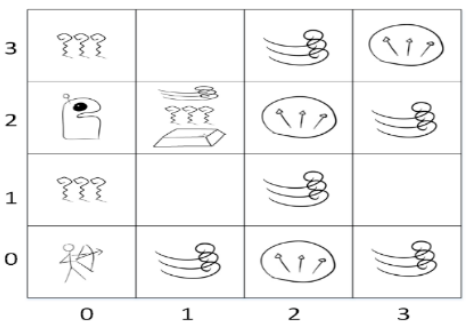

Figure 2: Elements of the Wumpus World

Environment shown as Figure 1 and Table 1:

$-4 * 4$ grid;

- Agent starts at $[0,0]$

-One gold and one wumpus are uniformly randomly located except $[0,0]$;

-Any square except $[0,0]$ can be a pit with probability of 0.2 . Sensors :

-Stench when adjacent to the wumpus;

-Breeze when adjacent to a pit;

-Glitter when reaching the gold; 
-Agent won't walk into a wall;

-Scream when the wumpus dies.

\subsection{Propositional Logic Representation}

Environment expressed by propositional logic;

-syntax defines the sentence structures in the language;

-semantics defines the meanings of sentences;

-we use the term model in place of the possible world (p-pit, w-wumpus, g-gold,

s-stench, b-breeze);

-KB-— knowledge base :

--64 symbols: $\mathrm{W}_{0,0}-\mathrm{W}_{3,3} ; \mathrm{P}_{0,0}-\mathrm{P}_{3,3} ; \mathrm{S}_{0,0}-\mathrm{S}_{3,3} ; \mathrm{B}_{0,0}-\mathrm{B}_{3,3} ;$

--physical rules included in knowledge base:

(1) $\mathrm{B}_{\mathrm{X}, \mathrm{Y}} \Leftrightarrow\left(\mathrm{P}_{\mathrm{X}+1, \mathrm{Y}} \vee \mathrm{P}_{\mathrm{X}-1, \mathrm{Y}} \vee \mathrm{P}_{\mathrm{X}, \mathrm{Y}+1} \vee \mathrm{P}_{\mathrm{X}, \mathrm{Y}-1}\right)$;

(2) $S_{X, Y} \Leftrightarrow\left(W_{X+1, Y} V W_{X-1, Y} V W_{X, Y+1} V W_{X, Y-1}\right)$;

(3) $\mathrm{W}_{0,0} \vee W_{0,1} \vee W_{0,2} \vee \ldots \vee W_{3,3}$;

(4) $\neg W_{0,0} \vee \neg W_{0,1}, \neg W_{0,0} \vee \neg W_{0,2, \ldots,} \neg W_{3,2} \vee \neg W_{3,3}$;

(5) $\neg \mathrm{P}_{0,0}, \neg \mathrm{W}_{0,0}$;

-Entailment: Knowledge base $\mathbf{K B}$ entails sentence $\alpha$ if and only if $\alpha$ is true in all worlds where $\mathbf{K B}$ is true, denoted as:

$\mathrm{KB} \vDash \alpha$

(1)

-Theorem: $\alpha \vDash \beta$ iff $M(\alpha) \subseteq M(\beta)$.

--only if: $\alpha \models \beta \Rightarrow \forall m \in M(\alpha)$, $\beta$ is true in $\mathrm{m} \Leftrightarrow \forall \mathrm{m} \in \mathrm{M}(\alpha), \mathrm{m} \in \mathrm{M}(\beta) \Leftrightarrow \mathrm{M}(\alpha) \subset$ $\mathrm{M}(\beta)$.

--if: $\forall \mathrm{m} \in \mathrm{M}(\alpha), \mathrm{m} \in \mathrm{M}(\beta) \Leftrightarrow \forall \mathrm{m}$ where $\alpha$ is true, $\beta$ is true $\Leftrightarrow \alpha \vDash \beta$.

\section{THE REASONING ALGORITHM OF THE WUMPUS WORLD}

\subsection{Extension Rules' Introduction:}

-DEFINITION: Given a clause $\mathrm{C}$ and an atom set $\mathrm{AT}$ : $\mathrm{D}=\{\mathrm{CV}$ a, $\mathrm{CV}\urcorner \mathrm{a} \mid$ "a" is an atom, $\mathrm{a} \in \mathrm{AT}$, "a" and "ᄀa" does not appear in $\mathrm{C}\}$. The operation proceeding from $\mathrm{C}$ to $\mathrm{D}$ is the extension rule on C. D is the result of the extension rule [10].

-THEOREM: Given a set of clauses $\Phi$ with its set of atoms $M(\mid M \vDash m)$, if the clauses in $\Phi$ are all maximum terms on $M$, then $\Phi$ is unsatisfiable iff it contains $2^{\mathrm{m}}$ clauses [10].

\subsection{Introduction of Novel Extension Rule}

NER applies the function getMaxTerm to lexicographically-ordered maximum terms with $\mathrm{i}$ incrementing from 0 . Each time, the function determines whether the maximum term can be consisted by the set of clauses. Once the set of clauses fail to consist a maximum term, we can decide that it is satisfiable, NER returns "SAT"; the set of clauses consisting all the maximum terms is unratifiable, NER returns "UNSAT"

\subsection{The Advantages of Novel Extension Rule}

Algorithm NER is a reasoning algorithm based on extension rules. It has a high operation efficiency, and avoids the complex solving process of principle of inclusion-exclusion. We use conjunctive normal forms to express the environment and the physical rules in the new algorithm. For each gird beside the edge, the algorithm knows whether the gird is safe by judging whether the set of known conjunctive normal forms entails the conjunctive normal form that is no wumpus or pits, then plans a safe route.

\subsection{The New Algorithm}

We provide a new algorithm base on extension rules and NER:

Algorithm NER_WUMPUS_AGENT

(1) inputs: percept, a list, [stench, breeze, glitter]

(2) static: KB, initially containing the "physics" of the wumpus world

$x, y$, orientation, the agent's position (initially $[0,0]$ ) and

orient (initially north)

visited, an array indicating which squares have been

visited, initially false

action, the agent's most recent action, initially null

Plan, an action sequence, initially empty

(3) update: $x, y$, orientation, visited based on action

(4) if stench then insert (KB, $\left.\mathrm{S}_{\mathrm{x}, \mathrm{y}}\right)$

(5) if breeze then insert $\left(K B, B_{x, y}\right)$

(6) if glitter then action $\leftarrow$ grab

(7) else if plan is nonempty then action $\leftarrow$ pop (plan)

(8) else if for some fringe square $[i, j]$, NER $\left(K B, P_{i, j}, W_{i, j}\right)==U N S A T$

(9) then insert (KB, $\left.\neg \mathrm{P}_{\mathrm{i}, \mathrm{j}}\right)$

insert $\left(\mathrm{KB}, \neg \mathrm{W}_{\mathrm{i}, \mathrm{j}}\right)$

plan $\leftarrow([\mathrm{i}, \mathrm{j}]$, visited $)$

action $\leftarrow$ pop(plan)

(10) else action $\leftarrow$ a randomly choose to move

\section{EXPERIMENTAL RESULTS}

E.g.1: the agent can find the gold. 
The map from running is like Figure 3:

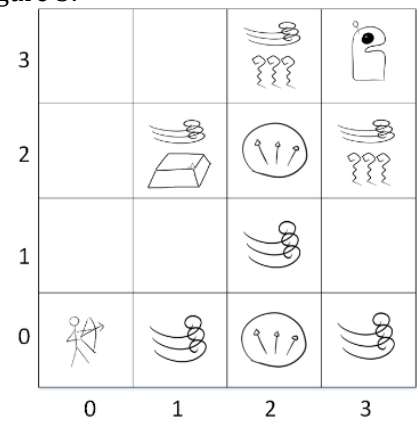

Figure 3: The map of experiment 1

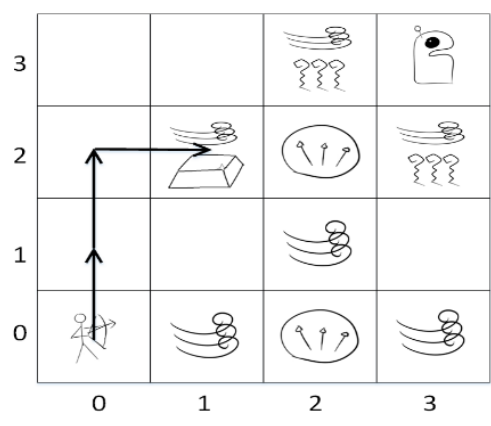

Figure 4: A feasible route of experiment 1

It's not hard to find a straight way to the gold in the map (Figure 4), the program running result is like Table 1:

Table 1: The result of experiment 1

\begin{tabular}{|l|l|l|l|}
\hline Position & Orientation & Destination & Results (position, state) \\
\hline$(0,0)$ & - & - & - \\
\hline$(0,0)$ & E & $(1,0)$ & $(1,0), \mathrm{B}$ \\
\hline$(1,0)$ & $\mathrm{S}$ & - & $(1,0), \mathrm{B}$ \\
\hline$(1,0)$ & $\mathrm{W}$ & $(0,0)$ & $(0,0)$ \\
\hline$(0,0)$ & $\mathrm{E}$ & $(1,0)$ & $(1,0), \mathrm{B}$ \\
\hline$(1,0)$ & $\mathrm{N}$ & $(1,1)$ & $(1,1)$ \\
\hline$(1,1)$ & $\mathrm{E}$ & $(0,1)$ & $(0,1)$ \\
\hline$(0,1)$ & $\mathrm{N}$ & $(1,1)$ & $(1,1)$ \\
\hline$(1,1)$ & $\mathrm{E}$ & $(2,1)$ & $(2,1), \mathrm{B}$ \\
\hline$(2,1)$ & $\mathrm{W}$ & $(1,1)$ & $(1,1)$ \\
\hline$(1,1)$ & $\mathrm{N}$ & $(1,2)$ & $(1,2), \mathrm{G}, \mathrm{B}$ \\
\hline
\end{tabular}

E.g.2: The agent can't make the right decision (agent may move into the pit)

-The map from running is like Figure 5:

-The program running result is like Table 2;

Table 2: The result of experiment 2

\begin{tabular}{|l|l|l|l|}
\hline Position & Orientation & Destination & $\begin{array}{l}\text { Results } \\
\text { (position,st } \\
\text { ate) }\end{array}$ \\
\hline$(0,0)$ & - & - & - \\
\hline$(0,0)$ & E & $(1,0)$ & $(1,0), \mathrm{B}$ \\
\hline$(1,0)$ & E & $(2,0)$ & $(2,0), \mathrm{B}$ \\
\hline$(2,0)$ & S & - & $(2,0), \mathrm{B}$ \\
\hline$(2,0)$ & W & $(1,0)$ & $(1,0), \mathrm{B}$ \\
\hline$(1,0)$ & N & $(1,1)$ & $(1,1), \mathrm{B}$ \\
\hline$(1,1)$ & E & - & $(1,1), \mathrm{B}$ \\
\hline$(1,1)$ & S & $(1,0)$ & $(1,0)$ \\
\hline$(1,0)$ & W & $(0,0)$ & $(0,0), \mathrm{B}$ \\
\hline$(0,0)$ & N & $(0,1)$ & $(0,1), \mathrm{P}$ \\
\hline
\end{tabular}

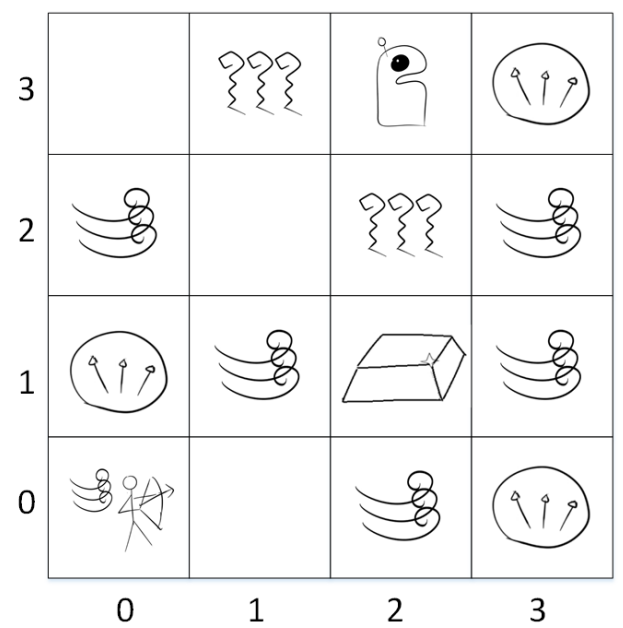


Figure 5: The map of experiment 2

According to the result of running, we can find that when agent move to $(0,2)$ and $(1,1)$, agent can know that in one or more parts of $(0,1),(2,1),(1,2),(3,1)$ may have the pits. It tells us that there doesn't have any position that is absolutely safe. Then agent will move to a random position. There agent may move into the pit.

E.g.3: The agent will die (it may around a position there are all pits, or wumpus and gold located in a same position, but the agent can't get the wumpus position according to the around position.)

-The map from running is like Figure 6;

-The program running result is like Table 3;

Agent has a sense of stench when it is in $(0,0)$, it knows that wumpus may located in $(1,0)$ or $(0,1)$. but can't know the absolutely position. In this way agent will move to a random position.

\section{CONCLUSIONS}

The developing trend of artificial intelligence must be letting the robot have cognitive reasoning capability and can adapt to the complex and changeable environment. In this paper, Wumpus World is an example to apply Extension Rule to agent's reasoning which shows the great potential Extension Rule has. With the development of the robot cognitive reasoning, Extension Rule, as an inverse of resolution, will be wildly used in the field of artificial intelligence.

Table 3: The result of experiment 3

\begin{tabular}{|l|l|l|l|}
\hline Position & Orientation & Destination & $\begin{array}{l}\text { Results } \\
\text { (position,state) }\end{array}$ \\
\hline$(0,0)$ & - & - & - \\
\hline$(0,0)$ & E & $(1,0)$ & $(1,0), \mathrm{W}, \mathrm{B}$ \\
\hline
\end{tabular}

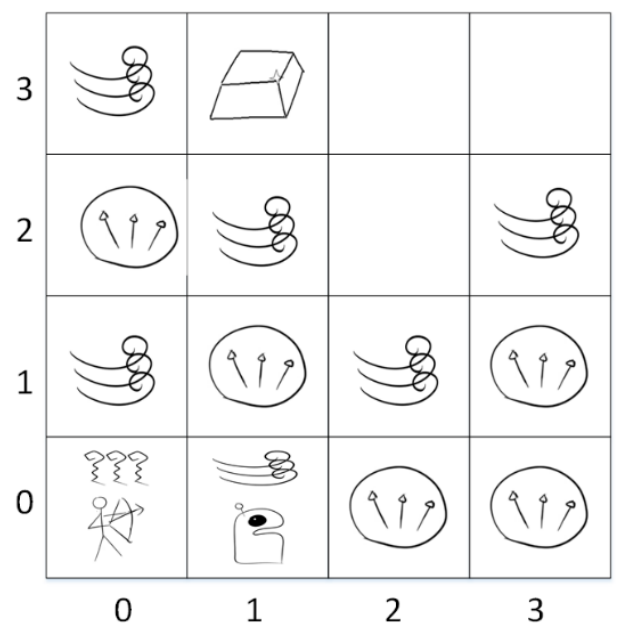

Figure 6: The map of experiment 3

\section{ACKNOWLEDGEMENT}

The work was supported by the National Nature Science Foundation of China under Grant Nos.61300049, 61502197, 61503044, the Specialized Research Fund for the Doctoral Program of Higher Education of China under Grant No.20120061120059, the China Postdoctoral Science Foundation under Grant No.2011M500612, the Key Program for Science and Technology Development of Jilin Province of China under Grant No.20130206052GX, the Natural Science Research Foundation of Jilin Province of China under Grant Nos.20140520069JH, 20150520058JH, 20150101054JC, the Graduate Innovation Fund of Jilin University under Grant No.2016181.

\section{REFERENCES}

[1] Goerick, C., Körner, E. 2007. Elements of Cognitive Robotics (Elemente kognitiver Robotik), De Gruyter. Oldenbourg Wissesnschaftsverlag, 49 (4), 213 217.

[2] Yu, Q.X., Hai, Y.M. 2009. Cognitive Robotics: A Survey. CIAC.

[3] Williams, A.B., Touretzky, D.S., Thompson, E.J.T., Manning, L.V., Boonthum, C., Allen, C.S. 2008. Introducing an experimental cognitive robotics curriculum at historically black colleges and universities. ACM SIGCSE Bulletin, 498-502.

[4] Vasile, C., Pavel, A., Buiu, C. 2010. Chidori A bio-inspired cognitive architecture for collective robotics applications. IFAC Proceedings, 43 (22), $40-45$. 
[5] Wei, G.W., Rui, W., Juan, L.X. 2016. Modeling and Validation of Cognitive Robot Control Behavior Using Uppaal. Journal of Chinese Computer Systems, (06), 1279-1283.

[6] Lin, H., Sun, J.G., Zhang, Y.M. 2003. Theorem proving based on extension rule. Journal of Automated Reasoning, 31, 11-21.

[7] Jigui, S., Ying, L., Xingjun, Z., Shuai, L. 2009. A Novel Theorem Proving Algorithm Based on Extension Rule. Journal of Computer Research and Development, 46 (1), 9-14.

[8] Jinyan, W., Wenxiang, G.U., Shaohua, Q.I.N., Extension rule: a survey. CAAI Transactions on Intelligent Systems, 9 (1), 1-11.

[9] Zixing, C. 2002. Agent-A New Research Topic for DAI. Computer Science, (12), 123-126.

[10] Wu, X., Sun, J., Hou, K. 2006. An Extension Rule Based First-Order Theorem Prover. In: Lang J., Lin F., Wang J. (eds) Knowledge Science, Engineering and Management. KSEM 2006. Lecture Notes in Computer Science, 4092 